\title{
Impact of Seizures and Anti-epileptic Drug Use on Survival in Patients with World Health Organization Grade II Glioma
}

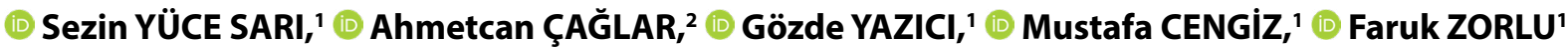

'Department of Radiation Oncology, Hacettepe University Faculty of Medicine, Ankara-Turkey

${ }^{2}$ Department of Radiotherapy, Hatay State Hospital, Hatay-Turkey

\begin{abstract}
OBJECTIVE
Epileptic seizures are prognostic for low-grade gliomas. However, the impact of anti-epileptic drug use (AED) on outcomes is unclear. We aimed to evaluate the impact of epileptic seizures and AED use on tumor control and survival rates after radiotherapy (RT) in patients with World Health Organization Grade II glioma.
\end{abstract}

\section{METHODS}

A total of 182 patients who underwent RT either early after surgery or in case of progression were retrospectively evaluated. The primary end point was the impact of seizures on overall survival (OS), local control (LC), and progression-free survival (PFS) rates. The secondary end point was the impact of AED use on these rates.

\begin{abstract}
RESULTS
Median follow-up was 80 months. The number of patients with epileptic seizures at the time of diagnosis was 113 (62\%). The 2-, 5-, and 10-year OS, LC, and PFS rates were significantly higher in patients with seizures $(\mathrm{p}=0.022$ and $\mathrm{p}=0.001$, respectively). When patients that did and did not use AED were compared, OS, LC, and PFS rates were similar. Besides, using AEDs in patients without seizures did not affect the OS, LC, and PFS rates. Carbamazepine use was associated with higher OS and PFS rates with a non-significant increase in LC. Phenytoin use yielded an increase in the PFS and LC rates in the first 2 years which turned to opposite after the $6^{\text {th }}$ year.
\end{abstract}

\section{CONCLUSION}

Seizures at the time of diagnosis and using AEDs for the treatment of seizures both increase survival rates in patients with Grade II glioma. Patients using carbamazepine had increased survival rates.

Keywords: Anti-epileptic drug; epilepsy; glioma; radiotherapy.

Copyright $\odot$ 2021, Turkish Society for Radiation Oncology

\section{Introduction}

Astrocytoma, oligodendroglioma, and mixed oligoastrocytoma were classified as World Health Organization (WHO) Grade II gliomas in 2007.[1] There is a lack of data on optimal treatment of Grade II gliomas. The majority of patients undergo surgery; however, due to the diffusely infiltrative nature of these tumors, total resection with an adequate margin is rarely achieved. Adjuvant radiotherapy (RT) is indicated in patients 
with residual tumor or progression following surgery. Although median overall survival (OS) is similar when $\mathrm{RT}$ is administered postoperatively and in case of progression, median progression-free survival (PFS) is higher and seizure control is better when RT is received earlier.[2] Furthermore, increasing the dose to $>45$ 50.4 Gy does not increase the OS and PFS rates. [3,4]

Epileptic seizure is one of the initial symptoms of brain tumors and can be observed in up to $85 \%$ of patients with low-grade gliomas.[5] The presence of seizures is a positive prognostic factor in gliomas, probably due to early diagnosis and treatment. $[6,7]$ The use of certain anti-epileptic drugs (AED) in the presence of seizures was also shown to increase the survival rate in patients with glioblastoma (GB).[8] However, the impact of using AED on survival in lowgrade gliomas is not yet clear.

In this context, we aimed to evaluate the outcomes of Grade II glioma patients diagnosed after epileptic seizures and compare them to patients without a seizure history. We also assessed whether there is an impact of the type of AED on local control (LC) and survival rates in these patients.

\section{Materials and Methods}

All patients underwent pretreatment physical examination, blood parameters, and magnetic resonance imaging (MRI) of the brain at the time of diagnosis. All patients had histologically proven WHO Grade II glioma according to the 2007 classification.[1] Patients under 18 years of age were excluded from the study, and only patients that received RT were included in the study. Patients that underwent biopsy solely had definitive RT. Patients that underwent subtotal resection (STR) or gross total resection (GTR) received adjuvant RT either shortly after surgery or when progression was observed.

Patients were treated with two-dimensional (2-D) RT before the year 2000, and with three-D conformal RT (CRT) or intensity-modulated RT (IMRT) after 2000. Treatments were administered through Theratron $780 \mathrm{C}$ or Theratron 1000 Cobalt-60 teletherapy devices (Best Theratronics Ltd, Canada), Philips SL25 (Philips Healthcare, Andover, MA, USA), Elekta Synergy Platform (Elekta AB, Stockholm, Sweden), or Varian Clinac DHX High performance (Varian Medical Systems Inc., Palo Alto, CA, USA) linear accelerators. The gross target volume (GTV) was defined as the tumor bed including the residual tumor if present in operated patients and the primary tumor at the time of diagnosis in T2weighted or FLAIR images on MRI. The clinical target volume (CTV)-1 was defined as GTV $+2-\mathrm{cm}$ margin, and CTV-2 was defined as GTV $+1-\mathrm{cm}$ margin. The planning target volume was defined as CTV $+5 \mathrm{~mm}$.

All statistical analyses were performed using Statistical Package for the Social Sciences (SPSS) version 21.0 (SPSS Inc., Chicago, IL, USA). First, we evaluated the characteristics and outcomes of all patients, and analyzed OS, LC, and PFS. We defined LC as no relapse of the tumor in the operative bed or the primary site, OS as the time from the date of diagnosis to the last followup or death from any cause, and PFS as the time from the date of diagnosis to the date of first appearance of local recurrence or death from any cause, respectively. Survival analyses were carried out using the KaplanMeier method and compared using the log-rank test. Age ( $<40$ vs. $\geq 40$ years), gender, Eastern Cooperative Oncology Group Performance Status (ECOG PS) (0-1 vs. $\geq 2$ ), comorbidity status, headache, sensorial deficit, motor deficit, cognitive deficit, nausea and vomiting $(\mathrm{N} \& \mathrm{~V})$, epileptic seizures, tumor location (frontal vs. parietal vs. temporal vs. occipital vs. thalamic vs. cerebellar), resection type (biopsy vs. STR vs. GTR), histopathology (pure oligodendroglioma vs. astrocytic component), tumor size at diagnosis $(<6 \mathrm{~cm}$ vs. $\geq 6 \mathrm{~cm}$ ), postresection tumor size ( $<3 \mathrm{~cm}$ vs. $\geq 3 \mathrm{~cm}$ ), RT indication (definitive vs. adjuvant), RT timing (definitive vs. early adjuvant vs. at progression), RT technique (2-D vs. 3 -D CRT vs. IMRT), total RT dose ( $<54$ Gy vs. $\geq 54$ Gy), and concurrent temozolomide use were included in the univariate analysis. The Cox proportional hazards model was used for the multivariate analysis. The potentially significant covariates following univariate analyses with significant contribution to the survival estimation $(\mathrm{p}<0.10)$ were preserved in the final multivariate model. Hazard ratios with $95 \%$ confidence interval (CI) were reported. $\mathrm{P}<0.05$ was considered significant.

Then, we divided all patients into two groups as having had or not having had seizures at the time of diagnosis and compared their characteristics and outcomes. The primary end point was the comparison of OS, LC, and PFS rates between patients with and without epileptic seizures at the time of diagnosis. The secondary end point was the comparison of OS, LC, and PFS in patients that did and did not use AED. We also evaluated the impact of individual AEDs on survival and LC rates. With this aim, we investigated the four most commonly prescribed AEDs which were valproic acid (VA), levetiracetam, phenytoin, and carbamazepine. Other AEDs were not analyzed as the number of patients using them was too small. Same statistical methods were used. 


\section{Results}

\section{Analysis of the Whole Population}

Medical charts of 182 patients diagnosed between June 1990 and September 2015 were retrospectively evaluated. Median age was 40 years (range: 19-70 years). Ninety-three (51\%) patients were female, and 89 (49\%) were male. Epileptic seizures were present in $113(62 \%)$ patients at the time of diagnosis. Out of these, $72(64 \%)$ had generalized and 41 (36\%) had partial seizures, respectively. Other symptoms were headache in $80(44 \%)$, motor deficit in $32(18 \%)$, sensory deficit in $30(17 \%)$, cognitive dysfunction in 19 $(10 \%)$, and $\mathrm{N} \& \mathrm{~V}$ in 16 (9\%) patients, respectively. Median tumor size was $55 \mathrm{~mm}$ (range: 6-130 $\mathrm{mm}$ ) at diagnosis and $30 \mathrm{~mm}(0-100 \mathrm{~mm})$ postoperatively, respectively. All patients were treated with RT either definitively or adjuvantly between March 1994 and November 2015. Median total dose was 54 Gy (range: 40-64 Gy) in median 27 fractions (range: 15-32). CTV-1 received a median 40 Gy (range: 28.8-64 Gy), while CTV-2 received a median 14 Gy (range: 4-28 Gy). Nine (5\%) patients received concurrent temozolomide of $75 \mathrm{mg} / \mathrm{m} 2 /$ day at a maximum dose of 150 $\mathrm{mg}$ /day. Other patient, tumor and treatment characteristics are listed in Table 1.

Median follow-up was 80 months (range: 3-340 months). The final status of all patients (alive or deceased) was reached. In all patients, median OS was 95.5 months (95\% CI=85.8-105.4 months, standard error $[\mathrm{SE}]=8.67)$. The 2-, 5-, and 10-year OS rate was $90 \%, 71 \%$, and $39 \%$, respectively. In univariate analysis, higher ECOG PS, N\&V, no epileptic seizures, astrocytic component, and $\geq 6 \mathrm{~cm}$ tumor at diagnosis were significantly associated with a lower OS rate $(\mathrm{p}=0.018, \mathrm{p}=0.028, \mathrm{p}<0.001, \mathrm{p}<0.001$, and $\mathrm{p}=0.006$, respectively). Although not statistically significant, thalamic localization and resection less than GTR also decreased the OS rate $(\mathrm{p}=0.063$ and $\mathrm{p}=0.1$, respectively). In multivariate analysis, ECOG PS, epileptic seizures, histopathology, and tumor size at diagnosis were prognostic factors for OS (Table 2).

The final status of the disease (regressed, stable, or progressed) could not be reached in 12 patients. Among the other 170 patients, progression was observed in 110 (60\%). The 2-, 5-, and 10 -year LC rate was $82 \%, 59 \%$, and $35 \%$, respectively. At the last follow-up, the tumor was upgraded to Grade 3 in 8 (4\%), and Grade 4 in 6 (3\%) patients, respectively. Median PFS was 66 months (95\% CI=53.9-78.2 months, SE=6.2). The 2-, 5-, and 10year PFS rate was $80 \%, 55 \%$, and $27 \%$, respectively. In
Table 1 Patient, tumor, and treatment characteristics

Number of patients (\%)

\begin{tabular}{lc}
\hline ECOG PS & \\
0 & $116(64)$ \\
1 & $52(28)$ \\
2 & $12(7)$ \\
3 & $2(1)$ \\
Epileptic seizures at diagnosis & \\
Present & $113(62)$ \\
Absent & $69(38)$ \\
Tumor location* & \\
Frontal lobes & $115(63)$ \\
Temporal lobes & $71(39)$ \\
Parietal lobes & $57(31)$ \\
Occipital lobes & $10(6)$ \\
Thalamus & $12(7)$ \\
Midline & $15(8)$ \\
Cerebellum & $2(1)$ \\
Brain stalk & $5(3)$ \\
Type of surgery & \\
Biopsy only & $22(12)$ \\
STR & $105(58)$ \\
GTR & $55(30)$ \\
Histopathology & \\
Astrocytoma & $55(30)$ \\
Oligodendroglioma & $99(55)$ \\
Mixed oligoastrocytoma & $28(15)$
\end{tabular}

*Tumors with more than 1 part of the brain involvement were added in all parts. ECOG PS: Eastern Cooperative Oncology Group performance status; STR: Subtotal resection; GTR: Gross total resection

univariate analysis, older age, higher ECOG PS, N\&V, no epileptic seizures, thalamic localization, resection less than GTR, astrocytic component, $\geq 6 \mathrm{~cm}$ tumor size at diagnosis and definitive RT significantly decreased the PFS rate $(\mathrm{p}=0.042, \mathrm{p}=0.003, \mathrm{p}=0.019, \mathrm{p}=0.001$, $\mathrm{p}=0.039, \mathrm{p}=0.002, \mathrm{p}=0.007, \mathrm{p}<0.001$, and $\mathrm{p}=0.015$, respectively). In multivariate analysis, age, ECOG PS, epileptic seizures, histopathology, and tumor size at diagnosis were prognostic factors for PFS (Table 2).

\section{Comparison of Patients with and without Epileptic Seizures}

Patients with and without epileptic seizures at diagnosis were compared according to their characteristics. Patients $<40$ years of age and with frontal or parietal lobe tumors had applied with significantly more seizures compared to their counterparts. On the other hand, patients with thalamic or midline involvement had significantly lower rates of seizures at diagnosis. Other characteristics were similar (Table 3). 


\begin{tabular}{|c|c|c|c|}
\hline Table 2 & $\begin{array}{l}\text { Results of multivariate an } \\
\text { patient population }\end{array}$ & yses in the & hole \\
\hline & $\mathbf{R R}$ & $95 \% \mathrm{Cl}$ & $\mathbf{p}$ \\
\hline OS & & & \\
\hline ECOG Ps & & & \\
\hline $0-1$ & 1 & $1.08-4.5$ & 0.029 \\
\hline$\geq 2$ & 2.2 & & \\
\hline Epileptic sei & izures & & \\
\hline Absent & 1 & $1.4-3.35$ & $<0.001$ \\
\hline Present & 2.17 & & \\
\hline Histopathol & $\log y$ & & \\
\hline Oligo & 1 & $1.7-3.7$ & $<0.001$ \\
\hline Astro & 2.5 & & \\
\hline Tumor size a & at diagnosis $(\mathrm{cm})$ & & \\
\hline$<6$ & 1 & $1.26-2.87$ & 0.002 \\
\hline$\geq 6$ & 1.9 & & \\
\hline PFS & & & \\
\hline Age (yea & ears) & & \\
\hline$<40$ & 1 & $1.06-2.25$ & 0.025 \\
\hline$\geq 40$ & 1.5 & & \\
\hline ECOG PS & & & \\
\hline $0-1$ & 1 & $1.15-4.9$ & 0.019 \\
\hline$\geq 2$ & 2.4 & & \\
\hline Epileptic sei & izures & & \\
\hline Absent & 1 & $1.03-2.4$ & 0.034 \\
\hline Present & 1.6 & & \\
\hline Histopathol & $\log y$ & & \\
\hline Oligo & 1 & $1.24-2.6$ & 0.002 \\
\hline Astro & 1.8 & & \\
\hline Tumor size a & at diagnosis $(\mathrm{cm})$ & & \\
\hline$<6$ & 1 & $1.33-2.9$ & 0.001 \\
\hline$\geq 6$ & 1.97 & & \\
\hline
\end{tabular}

RR: Relative risk; Cl: Confidence interval; OS: Overall survival; ECOG PS: Eastern Cooperative Oncology Group performance status; PFS: Progressionfree survival

Median OS was 119.3 months (95\% CI=93.7-144.9 months, $\mathrm{SE}=13)$ and 67.7 months $(95 \% \mathrm{CI}=45.5-89.9$ months, $\mathrm{SE}=11.3$ ) in patients with and without seizures, respectively. The 2-, 5-, and 10 -year OS rate was $95 \%$, $80 \%$, and $50 \%$ in patients with seizures and $81 \%, 57 \%$, and $19 \%$ in patients without seizures, respectively $(\mathrm{p}<0.001)$. The $2-, 5-$, and 10 -year LC rate was $87 \%$, $67 \%$, and $39 \%$ in the presence, and $73 \%, 44 \%$, and $27 \%$ in the absence of seizures, respectively $(\mathrm{p}=0.022)$. Median PFS was 80.8 months (95\% CI=67.9-93.7 months, $\mathrm{SE}=6.6)$ and 43.1 months $(95 \% \mathrm{CI}=37.8-48.3$ months, $\mathrm{SE}=2.7)$ in patients with and without seizures, respectively. The 2-, 5-, and 10-year PFS rates were $87 \%$, $65 \%$, and $33 \%$ in patients with seizures, and $69 \%, 38 \%$, and $17 \%$ in patients without seizures, respectively $(p=0.001)$. Among the 113 patients with seizures, the
OS, LC, and PFS rates were similar between the patients with generalized and partial seizures $(p=0.53$, $\mathrm{p}=0.9$, and $\mathrm{p}=0.86$, respectively).

\section{Impact of Antiepileptic Use and Type of Antiepilep- tic Drugs}

Among the 113 patients with seizures at diagnosis, 101 (89\%) used one or more AED, whereas 12 (11\%) did not. The 2-, 5-, and 10-year OS, LC, and PFS rates did not significantly differ between patients that did and did not use AED ( $\mathrm{p}=0.08, \mathrm{p}=0.8$ and $\mathrm{p}=0.46$, respectively). Among the 69 patients without seizures at diagnosis, 48 (70\%) used prophylactic AED. When the OS, LC, and PFS rates were compared, no statistically significant difference was found between these patients $(\mathrm{p}=0.87, \mathrm{p}=0.67$, and $\mathrm{p}=0.89$, respectively).

When the effect of the type of AED on OS, LC, and PFS was investigated, using VA and levetiracetam did not have a significant impact. On the other hand, patients that used carbamazepine had significantly higher rates of OS and PFS (Fig. 1). The 2-, 5-, and 10-year OS rate was $91 \%, 78 \%$, and $46 \%$ with carbamazepine compared to $89 \%, 69 \%$, and $36 \%$ without ( $\mathrm{p}=0.04$ ). The respective rates for PFS were $89 \%, 63 \%$, and $34 \%$ with carbamazepine compared to $77 \%, 52 \%$, and $24 \%$ without $(\mathrm{p}=0.02)$. Using carbamazepine also increased the $\mathrm{LC}$ rates although not statistically significant $(\mathrm{p}=0.06)$. Besides, patients that used phenytoin had higher rates of PFS in the first 2 years of follow-up but after that, phenytoin starts to have a negative impact on PFS and this difference becomes statistically significant after the $6^{\text {th }}$ year (the 2-, 5-, and 10-year PFS rate was $82 \%, 54 \%$, and $17 \%$ with and $78 \%, 56 \%$, and $40 \%$ without phenytoin, respectively, $\mathrm{p}=0.019$ ) (Fig. 2). A similar survival curve was achieved with phenytoin when the LC rate was assessed, although not statistically significant (the respective rates were $83 \%, 58 \%$, and $25 \%$ with and $81 \%, 60 \%$, and $46 \%$ without phenytoin, respectively, $\mathrm{p}=0.06)$. The effect of phenytoin use on OS rate was sparse $(\mathrm{p}=0.1)$.

To evaluate the individual effect of each AED, we separately analyzed the 96 patients that used monotherapy. The number of patients that used VA, carbamazepine, levetiracetam, and phenytoin solely was $2(2 \%), 13(14 \%), 16(17 \%)$, and $65(67 \%)$, respectively. The OS, LC, and PFS rate was significantly different among these patients (Table 4). The OS rate were significantly lower with phenytoin compared to VA $(\mathrm{p}=0.03)$ and carbamazepine $(\mathrm{p}=0.001)$, the $\mathrm{LC}$ rate was significantly lower with phenytoin compared to carbamazepine ( $\mathrm{p}=0.006)$, and the PFS rate was significantly lower with phenytoin compared to VA $(p=0.047)$ 
Table 3 Comparison of patient, tumor, and treatment characteristics in patients with and without epileptic seizures at the time of diagnosis

\begin{tabular}{|c|c|}
\hline \multirow{2}{*}{$\begin{array}{c}\text { Epileptic seizure at } \\
\text { the time of diagnosis }\end{array}$} \\
\cline { 1 - 2 } Characteristic & Number of patients (\%) \\
\hline Present Absent & p
\end{tabular}

\begin{tabular}{c}
\hline Age (year) \\
$<40$ \\
$\geq 40$ \\
Gender \\
Female \\
Male \\
ECOG PS \\
$0-1$ \\
$\geq 2$
\end{tabular}

Diabetes mellitus

Present

Absent

Hypertension

Present

Absent

Frontal lobe

Involved

Not involved

Temporal lobe

Involved

Not involved

Parietal lobe

Involved

Not involved

Occipital lobe

Involved

Not involved

Thalamus

Involved

Not involved

Midline

Involved

Not involved

Cerebellum

Involved

Not involved

Brain stem

Involved

Not involved

Resection width

Biopsy only

STR

GTR

Astrocytoma component

Present

Absent
25 (28) 0.01 *

$63(72)$

$50(53)$

44 (47)

$53(57)$

40 (43)

0.15

$60(67)$

29 (33)

$105(63) \quad 61(43)$

0.13

$6(37) \quad 8(57)$

2 (40)

$3(60)$

$110(63)$

$66(37)$

$4(44) \quad 5(56)$

$108(63)$

64 (37)

78 (68)

$37(32)$

$35(52)$

$32(48)$

49 (69)

22 (31)

64 (58)

47 (42)

45 (79)

$12(21)$

68 (54)

57 (46)

$6(60) \quad 4(40)$

$107(62)$

$65(38)$

4 (33)

8 (67)

$109(64)$

61 (36)

5 (33)

$108(65)$

$10(67) \quad 0.02^{*}$

59 (35)

$0(0)$

5 (100)

$113(64)$

64 (36)

$2(40)$

$3(60)$

111 (63)

$66(37)$

$13(59)$

9 (41)

67 (64)

38 (36)

$33(60)$

$22(40)$

49 (59)

34 (41)

$64(65)$

0.14
Table 3 Cont.

Epileptic seizure at

the time of diagnosis

Number of patients (\%)

\begin{tabular}{|c|c|c|c|}
\hline Characteristic & Present & Absent & $\mathbf{p}$ \\
\hline \multicolumn{4}{|l|}{$\begin{array}{l}\text { Tumor size at } \\
\text { diagnosis }(\mathrm{cm})\end{array}$} \\
\hline$<6$ & $55(61)$ & $35(39)$ & 0.59 \\
\hline$\geq 6$ & $47(65)$ & $25(35)$ & \\
\hline \multicolumn{4}{|l|}{$\begin{array}{l}\text { Postoperative tumor } \\
\text { size }(\mathrm{cm})\end{array}$} \\
\hline$<3$ & $45(60)$ & $30(40)$ & 0.65 \\
\hline$\geq 3$ & $59(63)$ & $34(37)$ & \\
\hline \multicolumn{4}{|l|}{ RT setting } \\
\hline Definitive & $11(58)$ & $8(42)$ & 0.87 \\
\hline Adjuvant & $83(62)$ & $51(38)$ & \\
\hline At progression & $19(66)$ & $10(35)$ & \\
\hline \multicolumn{4}{|l|}{ RT technique } \\
\hline $2-D$ & $30(64)$ & $17(36)$ & 0.96 \\
\hline 3-D conformal & $70(61)$ & $44(39)$ & \\
\hline IMRT & $13(62)$ & $8(38)$ & \\
\hline \multicolumn{4}{|l|}{ Total RT dose (Gy) } \\
\hline$<54$ & $11(55)$ & $9(45)$ & 0.49 \\
\hline$\geq 54$ & $102(63)$ & $60(37)$ & \\
\hline \multicolumn{4}{|c|}{ Concurrent chemotherapy } \\
\hline Present & $6(67)$ & $3(33)$ & 0.77 \\
\hline Absent & $107(62)$ & $66(38)$ & \\
\hline \multicolumn{4}{|c|}{ Tumor upgrade after RT } \\
\hline Present & $8(57)$ & $6(43)$ & 0.45 \\
\hline Absent & $105(63)$ & $63(37)$ & \\
\hline
\end{tabular}

*Statistically significant. ECOG PS: Eastern Cooperative Oncology Group performance status; STR: Subtotal resection; GTR: Gross total resection; RT Radiotherapy; IMRT: Intensity modulated radiotherapy

and carbamazepine ( $\mathrm{p}=0.001$ ). However, the number of patients with a tumor $\geq 6 \mathrm{~cm}$ was significantly higher in patients that used phenytoin compared to patients using other drugs ( $\mathrm{p}=0.018$ ).

\section{Discussion}

An epileptic seizure is a common first clinical sign of a brain tumor. Although it deteriorates the patient's quality of life, it has been shown to be a favorable prognostic factor for both low- and high-grade gliomas. $[7,9]$ This may be due to the fact that the seizures lead to earlier diagnosis and thus, earlier treatment of the tumor.[10] In the literature, low-grade tumors have a higher incidence of seizures compared to high-grade tumors.[11] The risk of seizures is $70-90 \%$ in oligo- 

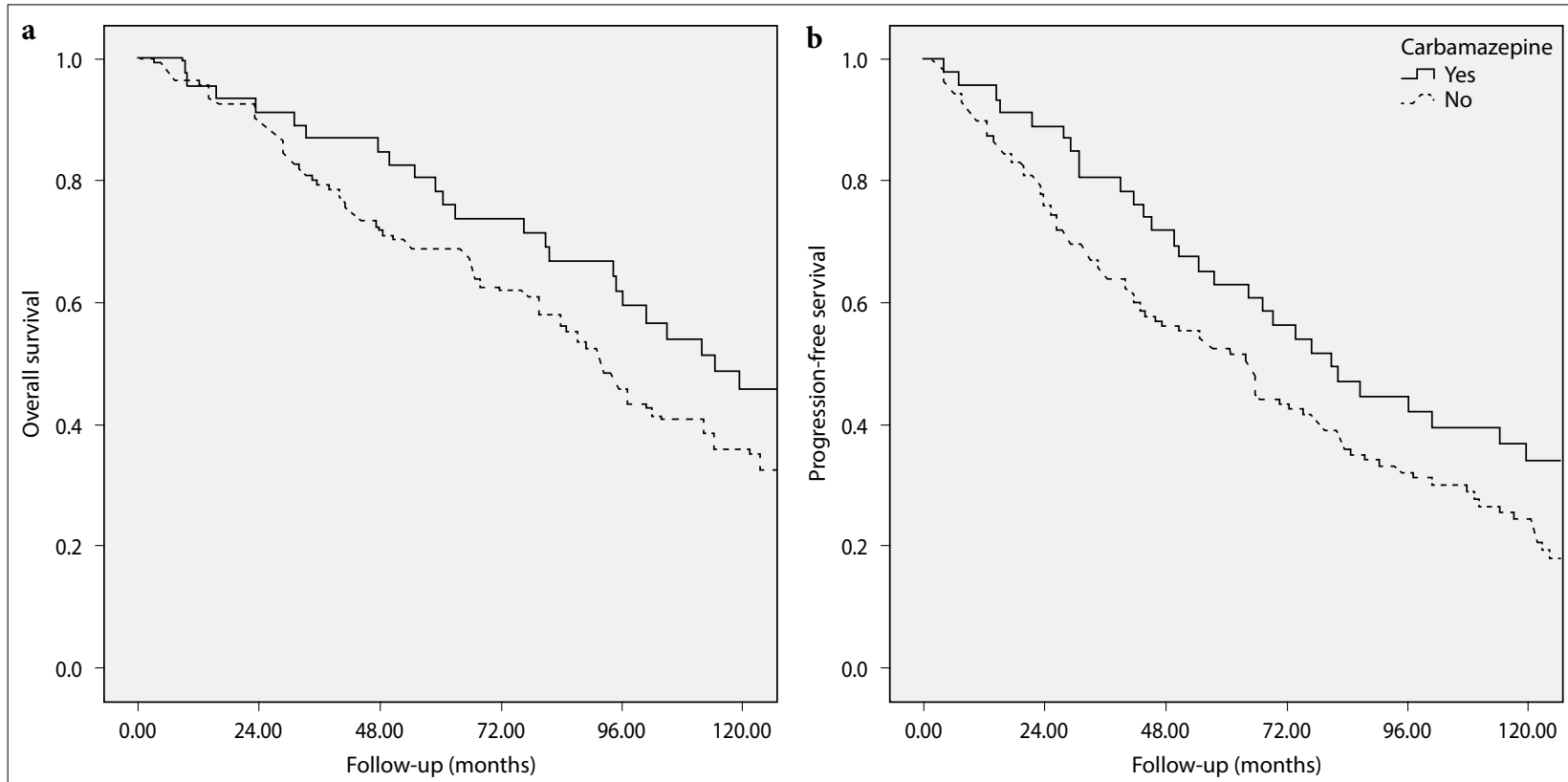

Fig. 1. Comparison of the (a) overall survival rate and (b) progression-free survival rate in patients that did and did not use carbamazepine. The differences are statistically significant after 2 years of follow-up $(\mathrm{p}=0.04$ and $\mathrm{p}=0.02$, respectively).

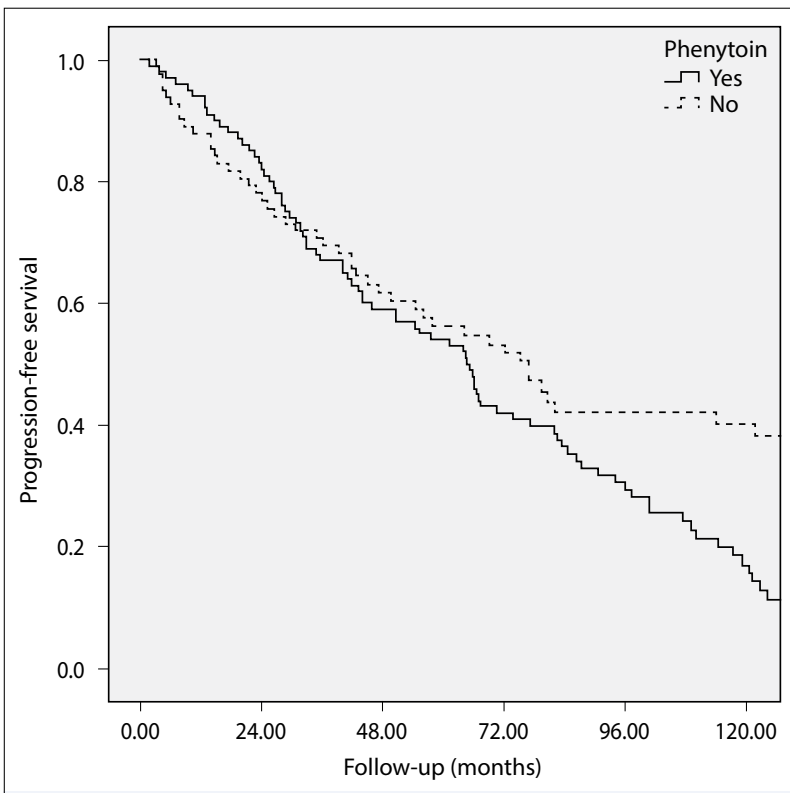

Fig. 2. Comparison of the progression-free survival rate in patients that did and did not use phenytoin.

dendroglioma, $60-85 \%$ in diffuse low-grade glioma, and $40-60 \%$ in GB. $[5,12]$ Other factors for increased risk of seizures are supratentorial location and oligodendroglial component. Seizures are most commonly observed in tumors of the frontal, temporal, and parietal lobes and are not typically observed with tumors of the deep structures, brainstem, or cerebellum.[13] When these characteristics are summoned, the risk of having epilepsy is higher in patients that are expected to survive longer. Therefore, control of seizures is an important issue in patients with Grade II glioma in terms of quality of life. Our finding of longer OS and PFS rates in Grade II glioma patients with seizures at diagnosis is consistent with these data. We found the rate of epileptic seizures at diagnosis $62 \%$, similar to the rate in the literature.[6,14] Besides, in our study, patients with frontal and parietal lobe tumors had a higher rate of seizures.

Epilepsy in patients with brain tumors is accepted as a partial epilepsy and can be with or without secondary generalized seizures. The incidence of generalized and partial seizures in brain tumors is $7-58 \%$ and $10-68 \%$, respectively, $[6,14]$ which was found $64 \%$ and $35 \%$ in our study. Seizures can be controlled by both anti-tumor treatment and AEDs. The choice of an appropriate AED depends on the type of epilepsy, as well as patient characteristics. The International League against Epilepsy recommends levetiracetam, carbamazepine, phenytoin, and zonisamide as level A AEDs, whereas VA is a level B AED.[15] Multiple AED with synergistic antiepileptic activity can be used in order to overcome pharmacoresistance.[16]

VA inhibits histone deacetylase, leads to an unfolding of the chromatin structure leaving the deoxyri- 


\begin{tabular}{|c|c|c|c|c|c|}
\hline & $\begin{array}{c}\text { Valproic acid } \\
\quad(n=2)\end{array}$ & $\begin{array}{c}\text { Carbamazepine } \\
\qquad(n=13)\end{array}$ & $\begin{array}{l}\text { Phenytoin } \\
(n=65)\end{array}$ & $\begin{array}{l}\text { Levetiracetam } \\
\quad(n=16)\end{array}$ & $\mathbf{p}$ \\
\hline \multicolumn{6}{|c|}{ OS y (\%) } \\
\hline 1 & 100 & 100 & 100 & 94 & 0.001 \\
\hline 2 & 100 & 100 & 97 & 94 & \\
\hline 5 & 100 & 85 & 71 & 94 & \\
\hline 10 & 100 & 77 & 33 & 56 & \\
\hline \multicolumn{6}{|l|}{ LCy (\%) } \\
\hline 1 & 100 & 100 & 94 & 81 & 0.018 \\
\hline 2 & 100 & 100 & 81 & 75 & \\
\hline 5 & 100 & 77 & 57 & 68 & \\
\hline 10 & 100 & 69 & 27 & 54 & \\
\hline \multicolumn{6}{|c|}{ PFS y (\%) } \\
\hline 1 & 100 & 100 & 94 & 81 & 0.002 \\
\hline 2 & 100 & 100 & 80 & 75 & \\
\hline 5 & 100 & 77 & 52 & 68 & \\
\hline 10 & 100 & 62 & 18 & 54 & \\
\hline
\end{tabular}

OS: Overall survival; LC: Local control; PFS: Progression-free survival

bonucleic acid (DNA) more susceptible to the effects of RT and chemotherapeutic drugs such as temozolomide.[17] The combination of VA and temozolomide was shown to induce apoptosis and autophagy of cancer cells in both in vitro and in vivo studies. $[18,19]$ VA has been shown to increase the survival rate in patients with GB when added to RT and temozolomide. [8] Levetiracetam also has an anti-tumor activity by inhibiting the transcription of the DNA repair protein methylguanine-methyl-transferase (MGMT) and increases the sensitivity of tumor cells to temozolomide.[20] In the retrospective study of Kim et al.,[21] levetiracetam significantly increased the median OS and PFS 9 months and 2.7 months, respectively, in patients with GB. However, the impact of these AEDs on survival in Grade II gliomas is not clear. In one retrospective study, the use of VA was shown to decrease the PFS rate in grade II and III gliomas, although not statistically significant.[22] Similarly, we did not find any significant effect of VA or levetiracetam on survival in our study.

Phenytoin and carbamazepine are level A AEDs and their impact on survival in primary brain tumors is unclear. A Danish retrospective study did not find any significant effect of phenytoin on survival in patients with GB.[23] In the only prospective study in patients with primary and metastatic brain tumors, carbamazepine use yielded significantly higher OS rates as did levetiracetam and VA.[24] Similarly, carbamazepine had a positive impact on OS and PFS in Grade II glioma patients in our study, particularly after a long follow- up. On the other hand, patients using phenytoin had significantly lower PFS rates after 2 years of follow-up when compared to patients not using this AED. This finding persisted when patients that used monotherapy were evaluated separately along with significantly lower rates of OS and LC. However, the number of patients with a larger tumor was significantly higher in patients that used phenytoin solely.

In a brain tumor patient without seizures, an AED can be initiated because it can reduce the risk of first seizure, prevent status epilepticus, and improve quality of life.[25] However, a 2008 meta-analysis did not show any clinical benefit of prophylactic AED use in glioma patients, and current guidelines recommend tapering and discontinuing AED postoperatively in glioma patients that never had a seizure. $[26,27]$ There is only one retrospective study that suggested a benefit of seizure prophylaxis in GB patients.[28] There are no published data on this issue in patients with low-grade gliomas. In our study, we found no positive impact of prophylactic AED use in Grade II glioma patients.

\section{Limitations of the Study}

The present study has potential limitations. The retrospective nature and relatively small number of patients precluded us from giving definitive conclusions about the impact of AEDs on survival and LC in Grade II gliomas. For the same reason, we could not analyze the effect of histopathological parameters such as the MGMT mutation which was shown to affect the sur- 
vival rates.[29] The impact of individual AEDs could not definitively be determined because many patients used multiple AEDs. On the other hand, this is a single-center study of standardized diagnostic and treatment protocols. To the best of our knowledge, this is the first study to investigate the impact of AEDs in Grade II gliomas.

\section{Conclusion}

Using AED prolongs survival in patients with seizures without affecting LC; however, AEDs do not add a survival benefit in patients without seizures. VA and levetiracetam did not increase survival rates in patients with Grade II glioma in this retrospective study, opposite to the findings in GB patients in the literature. On the other hand, we found a significant benefit of using carbamazepine on OS and PFS rates but not on LC. Besides, we observed that phenytoin use has a negative impact on PFS on long-term follow-up. However, these findings may be incidental and should be evaluated in prospective studies.

Peer-review: Externally peer-reviewed.

Conflict of Interest: The authors declare that they have no conflict of interest.

Ethics Committee Approval: This study has been carried out in accordance with The Declaration of Helsinki for experiments involving humans. Ethical approval was waived by the local Ethics Committee of Hacettepe University in view of the retrospective nature of the study and all the procedures being performed were part of the routine care.

Financial Support: This research did not receive any specific grant from funding agencies in the public, commercial, or not-for-profit sectors.

Authorship contributions: Concept - S.Y.S., A.Ç., G.Y., M.C., F.Z.; Design - S.Y.S., A.Ç., G.Y., M.C., F.Z.; Supervision - S.Y.S., G.Y.; Funding - S.Y.S., G.Y.; Materials - S.Y.S., A.Ç.; Data collection and/or processing - S.Y.S., A.Ç.; Data analysis and/or interpretation - S.Y.S., G.Y.; Literature search - S.Y.S., G.Y.; Writing - S.Y.S., G.Y.; Critical review - G.Y., M.C., F.Z.

\section{References}

1. Louis DN, Ohgaki H, Wiestler OD, Cavenee WK, Burger PC, Jouvet A, et al. World Health Organization histological classification of tumours of the central nervous system. Acta Neuropathol 2007;114(2):97-109.

2. van den Bent MJ, Afra D, de Witte O, Ben Hassel M,
Schraub S, Hoang-Xuan K, et al. Long-term efficacy of early versus delayed radiotherapy for low-grade astrocytoma and oligodendroglioma in adults: The EORTC 22845 randomised trial. Lancet 2005;366(9490):985-90.

3. Karim AB, Maat B, Hatlevoll R, Menten J, Rutten $\mathrm{EH}$, Thomas DG, et al. A randomized trial on doseresponse in radiation therapy of low-grade cerebral glioma: European Organization for Research and Treatment of Cancer (EORTC) study 22844. Int J Radiat Oncol Biol Phys 1996;36(3):549-56.

4. Shaw E, Arusell R, Scheithauer B, O'Fallon J, O'Neill $\mathrm{B}$, Dinapoli R, et al. prospective randomized trial of low-versus high-dose radiation therapy in adults with supratentorial low-grade glioma: initial report of a North Central Cancer Treatment Group/Radiation Therapy Oncology Group/Eastern Cooperative Oncology Group Study. J Clin Oncol 2002;20(9):2267-76.

5. Kahlenberg CA, Fadul CE, Roberts DW, Thadani VM, Bujarski KA, Scott RC, et al. Seizure prognosis of patients with low-grade tumors. Seizure 2012;21(7):540-5.

6. Chang EF, Potts MB, Keles GE, Lamborn KR, Chang SM, Barbaro NM, et al. Seizure characteristics and control following resection in 332 patients with lowgrade gliomas. J Neurosurg 2008;108(2):227-35.

7. Danfors T, Ribom D, Berntsson SG, Smits A. Epileptic seizures and survival in early disease of grade 2 gliomas. Eur J Neurol 2009;16(7):823-31.

8. Yuan Y, Xiang W, Qing M, Yanhui L, Jiewen L, Yunhe M. Survival analysis for valproic acid use in adult glioblastoma multiforme: a meta-analysis of individual patient data and a systematic review. Seizure 2014;23(10):830-5.

9. Chaichana KL, Parker SL, Olivi A, Quinones-Hinojosa A. Long-term seizure outcomes in adult patients undergoing primary resection of malignant brain astrocytomas. Clinical Article. J Neurosurg 2009;111(2):282-92.

10. Chaichana KL, Halthore AN, Parker SL, Olivi A, Weingart JD, Brem H, et al. Factors involved in maintaining prolonged functional independence following supratentorial glioblastoma resection. Clinical Article. J Neurosurg 2011;114(3):604-12.

11. Iuchi T, Hasegawa Y, Kawasaki K, Sakaida T. Epilepsy in patients with gliomas: incidence and control of seizures. J Clin Neurosci 2015;22(1):87-91.

12. Kerkhof M, Dielemans JC, van Breemen MS, Zwinkels $\mathrm{H}$, Walchenbach R, Taphoorn MJ, et al. Effect of valproic acid on seizure control and on survival in patients with glioblastoma multiforme. Neuro Oncol 2013;15(7):961-7.

13. Armstrong TS, Grant R, Gilbert MR, Lee JW, Norden AD. Epilepsy in glioma patients: mechanisms, management, and impact of anticonvulsant therapy. Neuro Oncol 2016;18(6):779-89. 
14. You G, Sha ZY, Yan W, Zhang W, Wang YZ, Li SW, et al. Seizure characteristics and outcomes in 508 Chinese adult patients undergoing primary resection of low-grade gliomas: a clinicopathological study. Neuro Oncol 2012;14(2):230-41.

15. Glauser T, Ben-Menachem E, Bourgeois B, Cnaan A, Guerreiro C, Kalviainen R, et al. Updated ILAE evidence review of antiepileptic drug efficacy and effectiveness as initial monotherapy for epileptic seizures and syndromes. Epilepsia 2013;54(3):551-63.

16. Brodie MJ, Sills GJ. Combining antiepileptic drugs-rational polytherapy? Seizure 2011;20(5):369-75.

17. Bangert A, Cristofanon S, Eckhardt I, Abhari BA, Kolodziej S, Hacker S, et al. Histone deacetylase inhibitors sensitize glioblastoma cells to TRAIL-induced apoptosis by c-myc-mediated downregulation of cFLIP. Oncogene 2012;31(44):4677-88.

18. Chen CH, Chang YJ, Ku MS, Chung KT, Yang JT. Enhancement of temozolomide-induced apoptosis by valproic acid in human glioma cell lines through Redox regulation. J Mol Med (Berl) 2011;89(3):303-15.

19. Van Nifterik KA, Van den Berg J, Slotman BJ, Lafleur MV, Sminia P, Stalpers LJ. Valproic acid sensitizes human glioma cells for temozolomide and gamma-radiation. J Neurooncol 2012;107(1):61-7.

20. Bobustuc GC, Baker CH, Limaye A, Jenkins WD, Pearl G, Avgeropoulos NG, et al. Levetiracetam enhances p53-Mediated MGMT inhibition and sensitizes glioblastoma cells to temozolomide. Neuro Oncol 2010;12(9):917-27.

21. Kim YH, Kim T, Joo JD, Han JH, Kim YJ, Kim IA, et al. Survival benefit of levetiracetam in patients treated with concomitant chemoradiotherapy and adjuvant chemotherapy with temozolomide for glioblastoma multiforme. Cancer 2015;121(17):2926-32.
22. Redjal N, Reinshagen C, Le A, Walcott BP, McDonnell E, Dietrich J, et al. Valproic acid, compared to other antiepileptic drugs, is associated with improved overall and progression-free survival in glioblastoma but worse outcome in grade II/III gliomas treated with temozolomide. J Neurooncol 2016;127(3):505-14.

23. Knudsen-Baas KM, Engeland A, Gilhus NE, Storstein AM, Owe JF. Does the choice of antiepileptic drug affect survival in glioblastoma patients? J Neurooncol 2016;129(3):461-9.

24. Cacho-Diaz B, San-Juan D, Salmeron K, Boyzo C, Lorenzana-Mendoza N. Choice of antiepileptic drugs affects the outcome in cancer patients with seizures. Clin Transl Oncol 2018;20(12):1571-6.

25. Wychowski T, Wang H, Buniak L, Henry JC, Mohile N. Considerations in prophylaxis for tumor-associated epilepsy: prevention of status epilepticus and tolerability of newer generation AEDs. Clin Neurol Neurosurg 2013;115(11):2365-9.

26. Gross RA, Johnston KC. Levels of evidence: taking neurology to the next level. Neurology 2009;72(1):8-10.

27. Wu AS, Trinh VT, Suki D, Graham S, Forman A, Weinberg JS, et al. A prospective randomized trial of perioperative seizure prophylaxis in patients with intraparenchymal brain tumors. J Neurosurg 2013;118(4):873-83.

28. Liang S, Zhang J, Zhang S, Fu X. Epilepsy in adults with supratentorial glioblastoma: incidence and influence factors and prophylaxis in 184 patients. PLoS One 2016;11(7):e0158206.

29. Berendsen S, Varkila M, Kroonen J, Seute T, Snijders TJ, Kauw F, et al. Prognostic relevance of epilepsy at presentation in glioblastoma patients. Neuro Oncol 2016;18(5):700-6. 